\title{
Análise do perfil dos pacientes com acne vulgar que são atendidos no consultório de
}

\section{dermatologia}

\author{
Analysis of the profille of patients with acne vulgaris who are seen at the dermatology office \\ Análisis del perfil de los pacientes con acné vulgar atendidos en el consultorio de dermatologia
}

Recebido: 18/12/2021 | Revisado: 26/12/2021 | Aceito: 04/01/2022 | Publicado: 06/01/2022

Evandra Marielly Leite Nogueira Pinheiro
ORCID: https://orcid.org/0000-0002-9916-9249
Centro Universitário Uninovafapi, Brasil
E-mail: evandranogueira @ hotmail.com
Emanuelle Singlindi Nascimento Falcão
ORCID: https://orcid.org/0000-0002-9330-0373
Centro Universitário Uninovafapi, Brasil
E-mail: emanuellenascimento10@ hotmail.com
Kaddyja Maria Borges de Andrade
ORCID: https://orcid.org/0000-0002-8890-6565
Centro Universitário Uninovafapi, Brasil
E-mail: kaddyjaaandrade@ hotmail.com

\begin{abstract}
Resumo
Objetivo: Identificar o perfil dos pacientes com acne vulgar que procura atendimento no Consultório de Dermatologia no período de janeiro a novembro de 2021. Metodologia: Trata-se de um estudo descritivo e transversal de abordagem quantitativa. A amostra foi constituída por 313 pacientes. Os dados coletados foram registrados inicialmente na planilha Microsoft Excel versão 2010 Windows. A tabulação e realização dos testes estatísticos foram realizados através do programa IBM SPSS Statistcs 20.0. Resultados: O perfil dos pacientes com acne a maioria são do sexo feminino, idade aproximada de 18 a 20 anos, com quadro de acne inflamatória moderada, que se mantinham em tratamento com Isotretinoína e anticoncepcionais, a qualidade de vida dos pacientes entrevistados não teve concordância com a literatura, de modo que apresentou índices dos pacientes com acne criticamente baixos quando comparado aos índices da população sem acne. Conclusão: Este estudo permitiu identificar o perfil dos indivíduos com acne vulgar atendidos em um consultório. A acne é uma patologia de alta incidência que pode exercer impacto psicológico significativo, principalmente nos graus mais altos de severidade.
\end{abstract}

Palavras-chave: Acne Vulgar; Dermatologia; Perfil de saúde.

\begin{abstract}
Objective: To identify the profile of patients with acne vulgaris who seek care at the Dermatology Office from January to November 2021. Methodology: This is a descriptive and cross-sectional study with a quantitative approach. The sample consisted of 313 patients. The collected data were initially registered in the Microsoft Excel version 2010 Windows spreadsheet. The tabulation and performance of statistical tests were performed using the IBM SPSS Statistcs 20.0 program. Results: The profile of patients with acne, most of them are female, aged approximately 18 to 20 years, with moderate inflammatory acne, who remained under treatment with Isotretinoin and contraceptives, the quality of life of the interviewed patients was not in agreement. with the literature, so that it presented rates of patients with acne critically low when compared to the rates of the population without acne. Conclusion: This study allowed us to identify the profile of individuals with acne vulgaris seen in an office. Acne is a high-incidence pathology that can have a significant psychological impact, especially in the highest degrees of severity.
\end{abstract}

Keywords: Acne vulgaris; Dermatology; Health profile.

\begin{abstract}
o
Objetivo: Identificar el perfil de los pacientes con acné vulgar que buscan atención en el Consultorio de Dermatología de enero a noviembre de 2021. Metodología: Se trata de un estudio descriptivo, transversal con abordaje cuantitativo. La muestra estuvo formada por 313 pacientes. Los datos recopilados se registraron inicialmente en la hoja de cálculo de Windows de Microsoft Excel versión 2010. La tabulación y realización de las pruebas estadísticas se realizaron utilizando el programa IBM SPSS Statistcs 20.0. Resultados: El perfil de pacientes con acné, en su mayoría mujeres, de 18 a 20 años aproximadamente, con acné inflamatorio moderado, que permanecieron en tratamiento con Isotretinoína y anticonceptivos, la calidad de vida de las pacientes entrevistadas no estuvo de acuerdo. la literatura, de modo que presenta tasas de pacientes con acné críticamente bajas en comparación con las tasas de la población sin acné. Conclusión: Este estudio permitió identificar el perfil de las personas con acné vulgar atendidas en un
\end{abstract}


consultorio. El acné es una patología de alta incidencia que puede tener un impacto psicológico importante, especialmente en los grados más altos de gravedad.

Palabras clave: Acne vulgaris; Dermatología; Perfil de salud.

\section{Introdução}

A acne vulgar é a mais frequente doença cutânea, afetando 85 a 100\% da população em qualquer momento da vida. É caracterizada por pápulas foliculares não inflamatórias ou comedões e por pápulas inflamatórias, pústulas e nódulos, nas suas formas mais severas. Afeta as áreas da pele com maior densidade de folículos sebáceos, as quais incluem a face, a parte superior do tórax e o dorso (Silva et al., 2020).

O diagnóstico da acne vulgar é clínico, sendo geralmente simples distinguir as diferentes formas, comedônica, pápulopustulosa e nódulo cística. Sua terapêutica baseia-se no princípio do controle dos fatores que constituem sua patogenia, buscando assim: reduzir a produção sebácea e a queratinização folicular, diminuir a intensidade de colonização, principalmente, por Propionibacterium acnes e cessar a inflamação (Figueiredo et al., 2011).

A patogênese da acne ainda não é totalmente compreendida. Porém, quatro mecanismos (hiperqueratinização folicular, colonização por Propionibacterium acnes, produção de sebo e complexos mecanismos inflamatórios envolvendo o sistema imune inato e adaptativo) têm sido amplamente aceitos como processos subjacentes à acne. Suspeita-se que o Propionibacterium acnes possa ter um papel importante na patogênese da acne, pois causa dano tecidual através da liberação de várias enzimas, entre elas a lipase, que decompõe os triglicérides em glicerol e ácidos graxos livres (AGL), levando a um influxo de neutrófilos através da quimiotaxia (Zaenglein et al., 2016).

A acne vulgar é classificada, clinicamente, em: comedoniana, papulo-pustulosa, nodular cística e acne conglobata. Conforme a gravidade, acne é estadiada em leve, moderada ou grave. A acne comedoniana compreende lesões não inflamatórias envolvendo comedões abertos (conhecidos como cravos) e comedões fechados, com seborreia presente. Na acne papulo-pustular são encontradas lesões inflamatórias e não inflamatórias. As lesões inflamatórias superficiais são compostas de pápulas e pústulas. Lesões não inflamatórias constituem os comedões abertos e fechados. Na acne nodular são observados os nódulos que são lesões de consistência firme, dolorosas à palpação, com características inflamatórias e com diâmetro acima de 10mm (Nast et al., 2016).

O tratamento da acne é variável conforme o grau de acometimento. São muitas as opções terapêuticas para essa afecção cutânea, podendo ser empregadas terapias de modo isolado ou em combinação. O tratamento farmacológico é aplicado sob a forma tópica e/ou sistêmica, de forma a controlar o avanço e evitar sequelas que podem ser mais preocupantes num segundo momento. A maior parte dos tratamentos estéticos apresentam finalidade comedolítica, reduzem a seborreia e a inflamação e ainda eliminam ou melhoram a aparência das cicatrizes de acne (Figueredo et al., 2011).

Diante do exposto a problemática dessa pesquisa é: Qual o perfil dos pacientes acometidos por acne vulgar que são atendidos no ambulatório o de dermatologia?

Sendo assim, os objetivos da referente pesquisa foram: Analisar o perfil dos pacientes com acne vulgar atendidos no Consultório de Dermatologia no período de janeiro a dezembro de 2021, descrever o perfil do paciente com acne vulgar, conhecer como é realizada a diagnosticado o grau de evolução da acne vulgar no paciente atendido no consultório, identificar de que forma o dermatologista realiza o diagnostico referente a classificação da acne vulgar conforme a classificação da mesma. 


\section{Metodologia}

Trata-se de um estudo descritivo e transversal de abordagem quantitativa Segundo Silveira \& Córdova (2009) pesquisa quantitativa refere-se ao tipo de pesquisa que requer a análise de dados objetivos que sejam passíveis de quantificação e pressupõe a previsão de mensuração das variáveis preestabelecidas, almejando verificar e explicar sua influência sobre outras variáveis, a partir de uma análise de frequência de certas incidências e de correlações estatísticas que demonstrem o processo de causalidade entre as variáveis.

As pesquisas com abordagem descritiva têm como principal objetivo as características de determinada população ou fenômeno e até mesmo a relação entre as variáveis. São inúmeros os estudos que podem ser classificados como descritivos e uma das características mais comuns é a utilização de técnicas padronizadas de coleta de dados, tais como questionários e a observação sistemática (Gil, 2002).

A coleta de dados foi realizada em um consultório de dermatologia de uma instituição de caráter privado que realizam tratamento de pacientes com acne vulgar na cidade de Teresina-PI.

A amostra para o estudo foi constituída por 313 pacientes atendidos em um consultório de dermatologia de uma instituição privada, selecionados aleatoriamente entre os 1680 que frequentam o consultório de Dermatologia de janeiro a novembro. Esse número foi calculado através da fórmula $\mathrm{n}=\left(\mathrm{z}^{2} \cdot 0,25 \cdot \mathrm{N}\right) /\left(\mathrm{E}^{2}(\mathrm{~N}-1)+\mathrm{Z}^{2} \cdot 0,25\right)=$ $\left(1,96^{2} \cdot 0,25 \cdot 1680\right) /\left(0,05^{2} \cdot 1679+1,96^{2} \cdot 0,25\right)$, na qual, z é o valor crítico, e a margem de erro e $\mathrm{N}$ o tamanho da população, considerando o grau de confiança de $95 \%(\mathrm{z}=1,96)$, margem de erro $\mathrm{E}=5 \%$ e $\mathrm{N}=1680$.

A coleta de dados foi realizada por meio de um questionário estruturado, sendo aplicado individualmente nos meses de setembro e outubro de 2021. Ressalta-se que a data da aplicação do questionário foi previamente agendada, respeitando a disponibilidade de tempo dos participantes e visando não inferir nas suas atividades. O tempo de duração da participação dos participantes nesta pesquisa durou em torno de trinta minutos.

Os critérios de inclusão para participar da pesquisa foi: pacientes diagnosticados com acne vulgar, que frequentam o consultório de dermatologia, com idade média de 18 (dezoito) à 45 (quarenta e cinco) anos. Foram excluídos do estudo participantes que sejam menores de 18 anos ou acima de 45 anos e que não tenham diagnóstico confirmado de acne vulgar.

Vale ressaltar que o acesso aos instrumentos de coleta de dados somente foi permitido após o participante ter acesso e concordar com o detalhamento da pesquisa, descrito no Termo de Consentimento Livre e Esclarecido-TCLE e declarar o aceite assinalando "li e concordo" na página do formulário.

Ressalta-se, ainda, que a coleta de dados foi realizada após a aprovação do projeto pelo Comitê de Ética do Centro Universitário UNINOVAFAPI.

Os dados coletados foram registrados inicialmente na planilha Microsoft Excel versão 2010 Windows. A tabulação e realização dos testes estatísticos foram realizados através do programa IBM SPSS Statistcs 20.0. Os resultados foram apresentados por meio de tabelas.

O projeto foi encaminhado à instituição coparticipantes da pesquisa, e após autorização, o projeto foi submetido à apreciação do Comitê de Ética e Pesquisa (CEP) do Centro Universitário UNINOVAFAPI, com o parecer de aprovado sob de número 4.952.037.

Os aspectos éticos dispostos na Resolução 466/12 do CNS foram garantidos por meio do Termo de Consentimento Livre e Esclarecido (TCLE), atendendo às exigências éticas e científicas fundamentais de uma pesquisa envolvendo seres humanos e, por meio do qual, os participantes serão esclarecidos sobre as etapas e os objetivos da pesquisa. 


\section{Resultados e Discussão}

Na pesquisa realizada foram entrevistados 313 pacientes com acne vulgar. A acne é uma das principais doenças de pele, a qual acomete pessoas de todo o mundo. De acordo com resultados obtidos na tabela 1 relacionado a faixa etária dos participantes. Observou-se que os casos acne vulgar segundo faixa etária o maior número de casos foi com a idade de 18 anos obtendo um equivalente de 11\% (51 participantes), seguido da faixa etária de 20 anos com 10,2\% (47 participantes).

Tabela 1 - Caracterização segundo faixa etária de pacientes com Acne vulgar. Teresina (PI), novembro de 2021.

\begin{tabular}{cccc}
\hline & Frequência & Percentual \\
\hline & 16 & 1 & 0,2 \\
18 & 51 & 11,0 \\
19 & 31 & 6,7 \\
20 & 47 & 10,2 \\
21 & 40 & 8,6 \\
22 & 34 & 7,3 \\
Idade & 37 & 8,0 \\
& 29 & 25 & 6,3 \\
& 24 & 22 & 5,4 \\
25 & 17 & 4,8 \\
26 & 27 & 3,7 \\
& 27 & 8 & 5,8 \\
& 28 & 13 & 1,7 \\
& 29 & 7 & 2,8 \\
& 30 & 10 & 1,5 \\
& 31 & 14 & 2,2 \\
& 12 & 38 & 3,0 \\
& 33 & 463 & 2,6 \\
& 34 & & 8,2 \\
& 35 & & 100,0 \\
\hline
\end{tabular}

Fonte: Pesquisa direta.

Os resultados da Tabela 1 podem ser justificados de acordo os estudos de Araújo et al. (2018) no qual retratam a acne como uma doença tão comum que pode-se dizer que é quase universal durante a adolescência, sendo o pico, com $85 \%$ de jovens afetados entre os 12-24 anos. Os resultados do estudo corroboram que a prevalência da acne vulgar no serviço da dermatologia de uma instituição privada, é em torno de 11,94\%, sendo a média da faixa etária em torno de até 25 anos.

$\mathrm{Na}$ análise dos dados sociodemográficos, observamos que na questão da idade, nossa amostra corrobora com os dados apresentados na literatura, em que 50\% dos pacientes com acne se encaixavam entre 17-27 anos, sendo a mediana 20 anos. As pesquisas ressaltam que, praticamente, todas as pessoas entre 15-17 anos apresentaram um episódio de acne na sua vida e cerca de 64\% apresentam acne até os 29 anos (Bhate \& Williams, 2013).

$\mathrm{Na}$ Tabela 2 verifica-se os índices de acne vulgar de acordo com sexo, estado civil, renda familiar e nível de escolaridade. O gênero predominante foi o feminino, com 76,5\% de pacientes, seguido pelo masculino com 23,5\%. Em relação o estado civil que predominou foi o de solteiro com 73,0\% de participantes, e 17,7\% eram casados. Na renda familiar observou-se que 45, 8\% tinham até um salário mínimo, dados que se aproximam dos participantes de possuíam dois salários mínimos, resultando em 42,3\% dos participantes. De acordo com o nível de escolaridade a predominância foi de até o ensino médio com um índice de $66,1 \%$. 
Tabela 2 - Características sociodemográficas de pacientes com acne vulgar segundo o sexo, estado civil, renda familiar e nível de escolaridade. Teresina (PI), novembro de 2021.

\begin{tabular}{llcc}
\hline Variável & & Frequência & $\%$ \\
\hline \multirow{2}{*}{ Sexo } & Feminino & 354 & $76,5 \%$ \\
& Masculino & 109 & $23,5 \%$ \\
& & 1 & $0,2 \%$ \\
Estado Civil & Casada/o & 82 & $17,7 \%$ \\
& Companheira/o & 37 & $8,0 \%$ \\
& Separada/o ou Divorciada/o & 5 & $1,1 \%$ \\
& Solteira/o & 338 & $73,0 \%$ \\
Renda Familiar & Dois salário mínimos & 196 & $42,3 \%$ \\
& Três ou mais salário mínimo & 55 & $11,9 \%$ \\
& Um salário mínimo & 212 & $45,8 \%$ \\
\multirow{2}{*}{ Nível de Escolaridade } & Até o Ensino Fundamental & 50 & $10,8 \%$ \\
& Até o Ensino Médio & 306 & $66,1 \%$ \\
& Só lê e escreve & 1 & $0,2 \%$ \\
& Superior & 55 & $11,9 \%$ \\
& Superior Incompleto & 51 & $11,0 \%$ \\
\hline
\end{tabular}

Fonte: Pesquisa direta.

Esses dados podem ser comparados com um estudo que são observadas as características demográficas dos pacientes, constatando que o sexo feminino foi de $66,7 \%$, a média de idade da amostra foi de ,18 a 28 anos, e a média de idade de início da doença foi de 17,1 anos. Além disso, corroborando com o mesmo estudo notou-se que a maioria dos pacientes tinha ensino médio completo $(64 \%)$, baixa renda $(83 \%)$. A estrutura da população amostral coincide com o perfil dos pacientes atendidos na instituição. (Lorduy et al., 2021).

Na Tabela 3, é caracterizada pelo índice de participantes que já fizeram tratamento para acne vulgar. Dentro os participantes entrevistados 48,8\% realizaram o tratamento da acne vulgar e 27,6\% além de realizar o tratamento sabiam o tipo de medicação do tratamento realizado.

Tabela 3 - Caracterização do uso de medicações para tratamento da acne vulgar. Teresina (PI), novembro de 2021.

\begin{tabular}{llcc}
\hline & & Frequência & $\%$ \\
\hline Já fez ou faz uso de & Não & 109 & $23,5 \%$ \\
algum medicamento para & Sim & 226 & $48,8 \%$ \\
tratar acne & Sim, qual? & 128 & $27,6 \%$ \\
\hline
\end{tabular}

Fonte: Pesquisa direta.

Em um estudo é possível detalhar que a escolha do tratamento da acne se define a partir da sua classificação. Para a acne de grau mais baixo (mais leve) utilizam-se tratamentos tópicos e em grau mais elevado associam-se, também, medicação via oral. O principal objetivo do tratamento é controlar e tratar as lesões existentes, prevenir a formação de cicatrizes permanentes, limitar a duração da condição e minimizar a morbidade. O paciente deve ser devidamente informado que a melhora pode ser observada em 3 a 6 semanas e é importante ter adesão ao tratamento (Fox et al., 2016).

Na Tabela 4, demostra o índice das medicações utilizadas pelos pacientes em relação ao tratamento da acne vulgar a predominância foi com uso da Isotretinoína com 28,4\%, seguido do anticoncepcional com $16 \%$ dos pacientes, os demais tratamentos tiveram uma variável irrelevante.

A isotretinoína é geralmente reservada para a acne nódulo-cística severa com cicatrizes ou acne resistente a outras terapias. É o fármaco que interfere de forma mais completa nos mecanismos físiopatológicos da acne. A administração de isotretinoína, por cerca de 20 semanas, resulta em cura clínica em aproximadamente $85 \%$ dos casos. O tratamento deve ser 
realizado com doses diárias de 0,5-1 $\mathrm{mg} / \mathrm{kg}$ por dia (em 2-3 tomadas/dia, após as refeições) ou um total de $120-150 \mathrm{mg} / \mathrm{kg}$ ao longo da duração do tratamento ( 6 a 8 meses). Para evitar exacerbação da doença, pode-se iniciar com uma dose mais baixa $(0,5 \mathrm{mg} / \mathrm{kg} / \mathrm{dia})$ durante o primeiro mês e depois aumentar para $1 \mathrm{mg} / \mathrm{kg} / \mathrm{dia}$ (Figueiredo et al., 2011).

Em outro estudo referência a isotretinoína como o inibidor mais eficaz da produção de sebo é a isotretinoína sistêmica (ácido 13-cis-retinóico), é derivado da vitamina A e possui ação via apoptose sebocitária, que não interfere no metabolismo do sebócito. É empregada nos casos de acne severa e refratária a outros tratamentos e em pacientes psicologicamente afetados devido a acne. Sua ação é antiqueratinizante, atrofiando as glândulas sebáceas gerando um efeito inflamatório da acne. Contudo, existem efeitos colaterais que podem surgir durante o tratamento, como: eritema; pele seca e/ou descamativa (recomenda-se empregar hidratantes) e pode ocorrer sensibilidade ao sol, necessitando de proteção com filtro solar (Melnik, 2018).

Esses dados se corroboram em outro estudo, confirmando que o anticoncepcional, é uma excelente escolha para as mulheres com agravamento pré-menstrual da acne, acne na idade adulta, acne envolvendo preferencialmente a metade inferior da face e pescoço, associada à seborreia; hirsutismo, e irregularidades do ciclo menstrual, com ou sem hiperandrogenismo, e para jovens sexualmente ativas com acne inflamatória. Os contraceptivos orais combinados (COCs), contendo um estrogênio [etinilestradiol (EE)] associado a um progestágeno de segunda geração (levonorgestrel, noretindrona) ou de terceira geração (desogestrel, norgestimato e gestodeno), ou a um antiandrogênio (acetato de clormadinona, ciproterona, dienogeste, trimegestona e drospirenona), são frequentemente prescritos (Silva \& Costa, 2014).

Tabela 4 - Caracterização da medicação utilizada pelos pacientes com acne vulgar. Teresina (PI), novembro de 2021.

\begin{tabular}{lrr}
\hline & Count & Table N \% \\
\hline Se sim, qual? & 128 & $27,6 \%$ \\
Anticoncepcional, Peróxido Benzoíla & 6 & $1,2 \%$ \\
Ácido Tópico (não soube informar) & 13 & $2,2 \%$ \\
Ácido Azelaico & 10 & $2,3 \%$ \\
Ácido Azelaico e Adapaleno & 6 & $1,2 \%$ \\
Peróxido de Benzoíla & 28 & $5,6 \%$ \\
Ácido Salić́lico & 6 & $1,2 \%$ \\
Ácido Salić́lico e Peróxido de Benzó́la & 2 & $0,4 \%$ \\
Adapaleno, Peróxido de Benzoíla e Ácido Azelaico & 2 & $0,4 \%$ \\
Amoxicilina & 6 & $1,3 \%$ \\
Anticoncepcional & 74 & $16,0 \%$ \\
Anticoncepcional, Ácido Salicílico & 6 & $1,2 \%$ \\
Anticoncepcional, Isotretinoína (Roacutan) & $0,4 \%$ \\
Anticoncepcional, Adapaleno + Peróxido de Benzoíla & 2 & $0,2 \%$ \\
Anticoncepcional, Ácido Azelaico & 1 & $0,4 \%$ \\
Anticoncepcional, Ácido Salicílico e Ácido Azelaico & 2 & $0,2 \%$ \\
Asepxia & 1 & $1,3 \%$ \\
Cremes & 6 & $0,2 \%$ \\
Adapaleno + Peróxido de Benzoíla & 1 & $4,3 \%$ \\
Adapaleno + Peróxido de Benzoíla, Ácido Azelaico e & 20 & $0,6 \%$ \\
Doxiciclina & 3 & $28,4 \%$ \\
Isotretinoína & 132 & $1,0 \%$ \\
Isotretinoína e Ácido Salicílico & 5 & $0,8 \%$ \\
Isotretinoína e Antibiótico & 4 & $0,4 \%$ \\
Isotretinoína Adapaleno + Peróxido de Benzoíla & 2 & $0,2 \%$ \\
Isotretinoína, Ácido Tópico e Antibiótico & 1 & $3,2 \%$ \\
Tretinoína & 2 & $0,4 \%$ \\
Tretinoína e Ácido Tópico & &
\end{tabular}

Fonte: Pesquisa direta.

$\mathrm{Na}$ Tabela 5, é possível detalhar a classificação do tipo de acne vulgar dos pacientes entrevistados, a prevalência da doença foi classificada no grau 2, com $54,4 \%$ dos participantes, seguindo do grau 1 com 38,2\%. Além disso, observou se os 
pacientes com o diagnóstico de acne vulgar se sentiam agressivo, frustrado ou envergonhado e $51 \%$ afirmaram que se sentiam um pouco. Ademais, foi questionado se a patologia tem interferido na vida social e nas atividades diárias, como também, em acontecimentos sociais ou na relação com indivíduos do sexo oposto e a prevalência que não, foi de $51 \%$. Em relação a frequentar outros espaços de entretenimento se causava desconforto, $69,8 \%$, responderam que não, sendo a predominância maior.

Ainda na Tabela 5, o item 5 referência como os entrevistados se sentiam em relação a aparência no último mês, e $54,2 \%$, não estavam preocupados. Já em relação a gravidade da acne vulgar a prevalência de 55,9 dos participantes foi que é um problema menor gravidade.

Tabela 5 - Caracterização segundo as classificações e qualidade de vida da acne vulgar. Teresina (PI), novembro de 2021.

\begin{tabular}{|c|c|c|c|}
\hline Variável & & Frequência & $\%$ \\
\hline \multirow{5}{*}{ Classificação da acne: } & Grau 1 & 177 & $38,2 \%$ \\
\hline & Grau 1, Grau 2 & 1 & $0,2 \%$ \\
\hline & Grau 2 & 252 & $54,4 \%$ \\
\hline & Grau 2, Grau 3 & 2 & $0,4 \%$ \\
\hline & Grau 3 & 31 & $6,7 \%$ \\
\hline \multirow{4}{*}{$\begin{array}{l}\text { Pelo fato de ter acne sentiu-se } \\
\text { agressivo, frustrado ou envergonhado }\end{array}$} & Muitíssimo & 31 & $6,7 \%$ \\
\hline & Muito & 78 & $16,8 \%$ \\
\hline & Nada & 115 & $24,8 \%$ \\
\hline & Um Pouco & 236 & $51,0 \%$ \\
\hline \multirow{4}{*}{$\begin{array}{l}\text { Acha que o fato de ter acne tem } \\
\text { interferido na sua vida social diária, } \\
\text { em acontecimentos sociais ou na } \\
\text { relação com indivíduos do sexo } \\
\text { oposto }\end{array}$} & Moderadamente, na maioria das atividades & 52 & $11,2 \%$ \\
\hline & Nada & 236 & $51,0 \%$ \\
\hline & Ocasionalmente ou apenas em algumas das atividades & 167 & $36,1 \%$ \\
\hline & Severamente, afetando todas as atividades & 8 & $1,7 \%$ \\
\hline \multirow{4}{*}{$\begin{array}{l}\text { Evita frequentar balneários, praias e } \\
\text { clubes ou usar roupas de banho por } \\
\text { causa da sua acne }\end{array}$} & A maior parte das vezes & 26 & $5,6 \%$ \\
\hline & Nunca & 323 & $69,8 \%$ \\
\hline & Ocasionalmente & 98 & $21,2 \%$ \\
\hline & Sempre & 13 & $2,8 \%$ \\
\hline \multirow{4}{*}{$\begin{array}{l}\text { Como descreveria ou o que sentiu } \\
\text { com a aparência da sua pele durante } \\
\text { o último mês }\end{array}$} & Geralmente preocupado & 57 & $12,3 \%$ \\
\hline & Muito deprimido e infeliz & 8 & $1,7 \%$ \\
\hline & Não preocupado & 251 & $54,2 \%$ \\
\hline & Ocasionalmente preocupado & 147 & $31,7 \%$ \\
\hline \multirow{4}{*}{$\begin{array}{l}\text { Por favor, indique o que pensa } \\
\text { quanto a gravidade da sua acne no } \\
\text { momento: }\end{array}$} & É um problema grave & 41 & $8,9 \%$ \\
\hline & É um problema menor & 259 & $55,9 \%$ \\
\hline & Não é um problema & 162 & $35,0 \%$ \\
\hline & Não poderia estar pior & 1 & $0,2 \%$ \\
\hline
\end{tabular}

Fonte: Pesquisa direta.

De acordo com o número e o tipo das lesões, definem-se as formas clínicas ou graus da acne vulgar. O grau I é a forma mais leve, não inflamatória ou comedoniana, onde há a presença de comedões fechados ou abertos; o grau II é a acne inflamatória ou pápulopustulosa, que apresenta-se com pápulas e pústulas de conteúdo purulento, associadas aos comedões; o grau III , conhecido como acne nódulo-cística, surgem nódulos mais exuberantes; no grau IV ou acne conglobata, há formação de abscessos e fístulas; o grau V, também chamado de acne fulminans, caracteriza-se por febre, leucocitose, poliartralgia, eritema inflamatório ou necrose, hemorragia das lesões, além das alterações de graus III e IV (Owen, 2014).

$\mathrm{Na}$ amostra analisada observou-se uma pequena diferença nos índices de qualidade de vida, em que o grupo pesquisado teve uma estabilidade na melhora da qualidade e vida. Esse achado não teve concordância com a literatura, a qual 
apresentou índices dos pacientes com acne criticamente baixos quando comparado aos índices da população sem acne (Haroon et al., 2019). Alguns estudos alegam que o impacto psicossocial da acne é maior que o da alopécia e da psoríase. Esse resultado pode se justificar devido ao grau da acne encontrada no estudo que variou entre leve e moderado, assim como, as técnicas estéticas existentes, melhorando assim, a qualidade de vida dos entrevistados (Oliveira, Sobreira, Velosa, Correia, Filipe, 2017).

\section{Conclusão}

A acne é uma patologia de alta incidência que pode exercer impacto psicológico significativo, principalmente nos graus mais altos de severidade. Quando ocorre em sua forma inflamatória, pode causar dor e desconforto ao paciente, além de problemas na autoestima. A escolha da terapêutica depende da avaliação clínica do grau de severidade da acne e pode envolver a medicação tópica e/ou oral, visando alcançar a eficácia terapêutica e a segurança de uso.

Este estudo permitiu identificar o perfil dos indivíduos com acne vulgar atendidos no consultório de dermatologia, ressaltando características desta dermatose na população, além dos principais tratamentos empregados. O perfil dos pacientes atendidos com acne é, portanto, de pacientes do sexo feminino, idade aproximada de 18 a 20 anos, com quadro de acne inflamatória moderada, que se mantinham em tratamento com Isotretinoína e anticoncepcionais, a qualidade de vida dos pacientes entrevistados não teve concordância com a literatura, de modo que apresentou índices dos pacientes com acne criticamente baixos quando comparado aos índices da população sem acne. Constituiu, portanto, estudo pioneiro na fundação e serve de base para estudos futuro.

\section{Referências}

Araújo, A. R, Silva, F. S., Moreira, N. V., Araújo, B. H. A., Armanelli, A. P, \& Silva, A. C. R. (2018) A luz como forma de tratamento da acne vulgar: uma revisão sistemática. [Internet]. Fisioter. Bras. 19(2):202-209. https://pesquisa.bvsalud.org/portal/resource/pt/biblio-911287

Bhate, K., \& Williams, H. C. (2013) Epidemiology of Acne Vulgaris. British Journal of Dermatology.:3(168); 474-485.

Figueiredo, A., Massa, A., Picoto, A., Soares, A. P., Basto, A. S., Lopes, C., Resende, C., 7 Rebelo, C., Brandão, F. M., Pinto, G. M., Oliveira, H. S., Selores, M., Gonçalo, M., \& Bello, R. T. (2011) Avaliação e tratamento do doente com acne - Parte II: Tratamento tópico, sistémico e cirúrgico, tratamento da acne na grávida, algoritmo terapêutico. Revista Portuguesa de Clínica Geral. 27(1):66-76.

Fox, L., Csongradi, C., Aucamp, M., Plessis, J., \& Gerber, M. (2016) Treatment Modalities for Acne. Molecules.21:1-20.

Gil, A. C. (2002) Como elaborar projetos de pesquisas. (4a ed.) Editora: Atlas.

Haroon, M. Z., Alam, A., Ullah, I., Ali, R., Taimur, M. F., \& Raza, K. (2019) Quality Of Life And Depression Among Young Patients Suffering From Acne. Journal of Ayub Medical College, Abbottabad JAMC. 31(3): 436-440, 2019.

Lorduy, H. J. C., Cely, P., Rincón, C., \& Riveros, H. (2021) Perfil de resistencia a la tetraciclina de Cutibacterium acnes en pacientes con acné vulgar en un centro dermatológico de Colombia. Actas Dermo-Sifiliográficas. 112(10): 873-880

Melnik, B. C. (2018) Acne vulgaris: The metabolic syndrome of the pilosebaceous follicle. Clin Dermatologia. 36(1):29-40.

Nast, A, Dreno, B., Bettoli, V., Degitz, K., Erdmann, R., Finay, A. Y., Ganceviciene, R., Haedersdal, M, Layton, A., Lo’ pez-Estebaranz, J. L., Ochsendorf, F., Oprica, C., Rosumeck,S., Rzany, B., Sammain, A., Simonart, T., Veien, N. K., ivkovic, M.V. Z., Zouboulis, C. C., \& Gollnick, H. (2016) European evidencebased: S3 guideline for the treatment of acne. European Dermatology Forum: subcommittee "Acne". JEADV. 2016; 30:1-28.

Oliveira, J. M., Sobreira, G., Velosa, J., Correia, D. T., \& Filipe, P. (2017) Association of Isotretinoin With Depression and Suicide: A Review of Current Literature. Journal of Cutaneous Medicine and Surgery. 22(1): 58-64.

Owen, C. (2014) Oral isotretinoin therapy for acne vulgaris http://www.uptodate.com/contents/oral-isotretinoin-therapy-foracnevulgaris?source=search_result\&search=isotretinoina+e+qualidade+de+vida\&selectedTitle=9 1 50. Acesso: 14 de dezembro 2021 .

Silva, A. M. F., \& Costa, F. P. (2014) Acne vulgar: diagnóstico e manejo pelo médico de família e comunidade. Revista Brasileira de Medicina de Família e Comunidade. 2014; 9(30):54-63.

Silva, P. R. S., Souza, M. L. P., Sena, N. V., Alves, A. F. V., Pontes, L. M., Amarante, M. S. L. D., \& Brandão, B. J. F. (2014) Perfil epidemiológico dos pacientes com acne vulgar atendidos na BWS, BWS Journal. 2020; 3(e20070077): 1-7.

Silveira, D. T., \& Córdova, F. P. (2009) A pesquisa científica. Métodos de pesquisa. Plageder. cap. 2, 33-34.

Zaenglein, A. L. Pathy, A. L, Schlosser B J, Alikhan, A, Baldwin H. E, Berson, D. S, Bowe, W. P, \& Graber, E. M. (2016) Guidelines of care for the management of acne vulgaris. J Am Acad Dermatol. 74(5):945-73. 